\title{
Comprehensive analyses of syntactic foam behaviour in deepwater environment
}

\author{
François Grosjean ${ }^{1, *}$, Nadège Bouchonneau ${ }^{1,2}$, Dominique Choqueuse ${ }^{2}$ and Valérie \\ Sauvant-Moynot ${ }^{1}$ \\ ${ }^{1}$ IFP Lyon, Echangeur A7-Sortie Solaize, BP 3, 69360 Solaize, France \\ 2 Ifremer Centre de Brest, BP 70, 29280 Plouzané, France \\ *: Corresponding author : Grosjean F., email address : francois.grosjean@ifp.fr
}

\begin{abstract}
:
Over the past 10 years, numerous studies were performed to better understand the behaviour of the glass syntactic foams used as thermal insulation of pipes for deepwater production. The obtained results outlined some specific behaviour of polymeric syntactic foams reinforced by glass microballoons in service conditions: both water uptake and mechanical stress have a key impact on thermal properties. This article focuses first on the wet behaviour of glass syntactic foams. The effect of water is investigated to better model the nature of water diffusing in syntactic foams with and without a topcoat protection. Secondly, the effect of hydrostatic pressure on coated structure is addressed by using a confined compression test. As polymer material is bonded to the steel surface, it is not submitted to pure hydrostatic loading but to non-spherical loading in the vicinity of the pipe. The confined compression test is then chosen to represent these non-spherical loadings of material. The rupture of glass microballoons is monitored by acoustic emission for different matrices and attempts are made to quantify the resulting acoustic emission signals by comparison with prior tomography results. These experimental analyses provide a better understanding of the main factors affecting the functional properties of syntactic foams.
\end{abstract}




\section{Introduction}

Increasing demand for oil and optimistic estimation of reserves in deep water sustain the development of offshore deepwater fields. Above $80 \$ /$ baril, all the kinds of oil can be economically produced, even the shale oil [1]. For these reasons, the ultra deep water (3000m water depth) is becoming one of the next issues.

The production of oil in ultra deep water faces lots of challenges. Among others is flow assurance which requires the use of thermally managed systems [2]. Due to multiphase flow in flowlines and risers, and consequently possible wax and hydrates formation, thermal insulation of subsea pipelines has become increasingly important. This thermal insulation is used to maintain fluid temperature from subsea completion to floating platforms above a given temperature around $40^{\circ} \mathrm{C}$ to facilitate the flow.

One of the first approaches was to place insulation materials in the annulus of pipe-in-pipe systems. But these systems are expensive and heavy to install. Alternative systems were therefore developed, based on steel pipe surrounded by insulation materials without external steel pipe. In this case, the insulation, mainly based on polymeric material, must withstand the high pressure, water ingress, high internal and low external temperatures.

Several technologies were developed:

independent modules placed around the steel pipe (Fig. 1); the syntactic foam is then in contact with water on all its surface, on the one hand cold water on the outer surface on the other hand hot water near the steel surface. This system was used among others for the development of Girasol field [3]

multilayer coatings including syntactic foam layer for insulation (Fig. 2). In this case, only the external surface of the system is in contact with water. This solution was used for example for the insulation of Bonga production steel catenary riser [4].

Fig.1 (simple)

Fig.2 (simple)

Over past ten years, numerous studies were performed to better understand and discuss degradation mechanisms of syntactic foams due to combined effects of pressure, temperature gradient, and water ingress [5-8].

Nevertheless, main of the studies were conducted on small samples of material, often for convenience aspects. Some of them revealed astonishing behaviours of syntactic foams, for instance, the effect of the nature of water (sea water or natural water) [9], the detrimental effect of combined solicitations including pressure, temperature and water [10].

All these results outlined the need to have full-scale thermal testing protocols and facilities to study the behaviour of thermal insulation coating systems on lengths of pipes under simulated service conditions. Some experimental facilities were developed to directly measure thermal properties [11]. More recently, some full-scale prototype tests were modelled to address their global long term behaviour taken into account the water ingress and the highly non linear ageing behaviour of syntactic foams [12].

Nevertheless, some behaviours need to be more accurately understood to better describe and then model these systems. This paper will investigate two of these particular behaviours of syntactic foams: the first part addresses the behaviour of syntactic foams when aged in different environments; the second part investigates the foams' response under non-isotropic mechanical solicitations.

\section{Experimental}

\section{Materials}

The effect of ageing environment has been studied on a commercial epoxy syntactic foam hardened by an anhydride curing agent. This resin has been reinforced with sodium-borosilicate glass micro-balloons of density $0.38 \mathrm{~g} . \mathrm{cm}^{-3}$ at $55 \%$ volume fraction. Some pure epoxy/anhydride resin samples have been also manufactured at the laboratory. 
The effect of a top-coat on the ageing of the foam has been studied on epoxy syntactic foam samples embedded in pure resin (Fig. 3). To reduce the amount of water diffusing into the foam, the pure resin was a classical formulation used at lab, with well known properties and in particular low water uptake. This resin consisted of a mixture of difunctional epoxy resin (Diglycidyl ether of bisphenol A DGEBA) and an amine hardener (4,4' methylenebis(3-chloro-2,6-diethylaniline MCDEA) at stoichiometric ratio. This formulation has been chosen because its ageing characteristics and especially the diffusion coefficients of water are well known. The thickness of the epoxy coat was around $2 \mathrm{~mm}$ (Fig. 3).

Fig.3 (simple)

The mechanical testing has been conducted on foams with different matrices: Polypropylene (PP), Polyurethane (PU) and Epoxy (EP). The three foams were filled with glass micro-balloons of same density $0.38 \mathrm{~g} \mathrm{~cm}^{-3}$. The samples were machined in cylindrical form with diameter $10 \mathrm{~mm}$ and height 10mm (Fig. 4).

Fig.4 (simple)

\section{Experiments}

\section{Gravimetry}

Isothermal ageing tests were performed at $80^{\circ} \mathrm{C}$ in deionised water, in natural sea water and in relative humidity $90 \%$ in an oven. The sizes of the samples were $50 \times 50 \times 2 \mathrm{~mm} 3$. The water uptake in percentage (\%mass gain) was calculated following ASTM D570 (mass gain determined on blotted dry samples normalised by the initial mass).

\section{Compression test}

The non isotropic solicitation has been applied using a confined compression set-up (Fig. 5). The strain rate was the same for all experiments: $5.10^{-3} \mathrm{~mm} / \mathrm{min}$. For monotonic tests, the load was increased up to $25 \mathrm{MPa}$ for rigid matrix (epoxy) and $20 \mathrm{MPa}$ for soft matrices (PU, PP). The tests were performed at room temperature.

Fig.5 (simple)

\section{Results and discussion}

\section{Wet behaviour of syntactic foams}

The mass gain of pure epoxy-anhydride samples aged at $80^{\circ} \mathrm{C}$ in deionised water, in natural sea water and in relative humidity is presented on Fig. 6 . The sigmoid form of the curve reveals a two step phenomenon: the first step corresponds to the plasticization of the resin by water molecules, the second one is attributed to the degradation of the polymer. This kind of behaviour is generally represented by a Langmuir law, which takes into account both the diffusion into the material but also other phenomena like degradation which give these abnormal results. In the case of an epoxy hardened by an anhydride, the degradation in hot water classically occurs by hydrolysis of the ester functions [14]. The study of the degradation of the resin was nevertheless not the main issue of this paper. Thus we were focusing on the first step where the diffusion kinetics in the resin have been similar in both liquid environments, whereas the mass gain in relative humidity is as expected lower, and corresponds more or less to ninety percents of water uptake in liquid [15].

Fig.6 (simple)

Fig.7 (simple) 
The results on syntactic foams are more surprising (Fig. 7). The behaviour is very sensitive to the environment. First, concerning sea and deionised water, the weight uptake was the highest for deionised water, whereas the sea water induced a smaller weight increase. This could be explained by the water activity [13]. This parameter $a_{w}$ is the ratio between the vapour pressure of water in a media (for example in salted water) and the vapour pressure of pure water in the same conditions. It is related to the availability of water molecules for a process. Its value is 1 for deionised water and 0.75 for $\mathrm{NaCl}$ saturated solution.

Due to the chemical products created during ageing, and especially when water molecules segregate to the glass-matrix interface, there is a difference between the activity of water in the ageing solution $\left(a_{s}\right)$ and the activity of water inside the material $\left(a_{m}\right)$. This difference creates an osmotic pressure:

$$
\Pi=\frac{R T}{V_{m}} \ln \frac{a_{s}}{a_{m}} \quad \text { with } \vee_{\mathrm{m}} \text { molar volume of water. }
$$

This equation shows that a higher activity of the ageing solution leads to a higher osmotic pressure, inducing then a bigger interface gap and promoting a higher volume of incoming water.

The second surprising result concerns the water uptake in relative humidity. In this case, the weight gain is very low, not proportional to the humidity level and to the water uptake of the foam in liquid environment.

As insulation material is often protected by a pure polymer layer, these results lead to wondering what kind of ageing medium is in contact with foam. Indeed, as the coating layer acts as a membrane, the water diffuses through it at a molecular state. The question of the behaviour of the syntactic foam in relation to these diffusing molecules is then: is it equivalent to liquid or relative humidity environment?

To answer this question some prototype samples were manufactured to simulate a foam embedded in pure resin which protected it from a direct contact with the liquid. These samples were aged in hot water $\left(80^{\circ} \mathrm{C}\right)$ during around 400 days. The weight uptake curve (Fig. 8) shows a regular increase up to almost $4 \%$ during the test duration.

This test has been modelled using the sample geometry and the diffusion properties of each material determined by an inverse method from absorption results in similar conditions. The sample has been meshed with three-dimensional elements and the water absorption simulation has been based on two hypotheses: 1) the diffusion in pure epoxy follows a Fick law [16]; 2) the diffusion in syntactic foam follows a Langmuir law in the case of liquid water or a Fick law in the case of relative humidity.

The comparison between experimental results and the two simulated curves shows that the water absorption behaviour of the syntactic foam is related to ageing of foam in liquid water.

These results outlined that, even protected by a topcoat, the syntactic foams age like in liquid water. The topcoat could reduce the ageing kinetic by a limitation of the quantity of liquid medium, since the permeation rate of water through the top coat is governing the water diffusion to the foam, but the ageing mechanism will be the same with or without this topcoat.

Fig.8 (double)

\section{Behaviour under non-hydrostatic solicitation}

This part addresses the particular mechanical behaviour of a multilayer coating system (Fig. 2). A structure of this type is commonly composed of a steel pipe and a 5-layer insulating coating made with several material types: solid polymers thermoset and thermoplastic, syntactic foam, each layer having a different functionality. First of all, the steel surface is covered by an adhesive which links the coating layer to steel. This adhesive is a fusion bonded epoxy. When the coating material is polypropylene based, an intermediate layer is necessary to link epoxy material to PP. This function 
is fulfilled by a modified PP layer which can mix with the PP layer on the one hand and also chemically react with epoxy material on the other hand. The third layer is a PP pure layer between modified layer and PP syntactic foam material. The main layer is the syntactic foam layer, which is several tenth millimetres thick. Its main function is to provide insulation between hot steel tube and cold sea water. The last layer is a pure PP layer (around few millimetres thick) which protects the foam from external damage during installation or during service. It also limits the diffusion of water.

Other materials are also used to realise an insulation coating. The manufacture process varies in function of design and material but all these kinds of structures must sustain non isotropic solicitation under external pressure. Indeed, the modelling of an insulated pipe under hydrostatic pressure reveals that the Von Mises stress are not constant through the thickness, meaning that the loading is not purely hydrostatic (Fig. 9). Due to the link between the insulation material and the steel surface, the foam movement is restricted in circumferential and longitudinal directions.

Fig.9 (simple)

To compare the behaviour of syntactic foam with different matrices, a confined compression set-up has been used. This solicitation has been chosen to simulate the mechanical loading actually applied on the insulation coating when it is bonded onto the steel surface.

The Fig. 10 presents the compression curves of three syntactic foam materials, two with soft matrices (PP and PU) and one with rigid matrix (epoxy). These tests were conducted up to $250 \mathrm{MPa}$, obviously a very high loading towards service conditions, in order to know the overall behaviour of the material until rupture. After a first increase of load, one can observe that there is more or less a plateau which corresponds to the rupture of the microballoons. When all the balloons are broken, there is compaction of the material and the load increases rapidly.

Fig.10 (simple)

The acquisition of acoustic emission signals during these mechanical tests gives more information [18].

Fig. 11 presents the evolution of cumulative number of hits and absolute energy of acoustic signals versus load for each kind of syntactic foam. As the three graphs have the same scale, it is easy to compare the behaviour of each material. First of all, the soft matrices lead to a progressive damage with a start at a very low load for PU resin. PP foam shows a very low activity and very low cumulative energy. Rigid matrix leads to very differing behaviour. There is quite no noise up to a relatively high load then, there is a sudden and great emission. As acoustic emission record needs the use of a threshold to eliminate the background noise, some low energy events are obviously missed but the comparison is relevant.

Above-mentioned differences can be explained by other observations performed by X-Ray tomography on same materials during mechanical compression using confined test [17]. This study reported qualitative and quantitative study of broken microballoons at different levels of damage (interrupted tests at increasing load level). For soft matrices, the damage occurred progressively and the first broken balloons were the biggest. For rigid epoxy matrix, there was no damage up to a load limit (around $100 \mathrm{MPa}$ ), above which the damage occurred massively and through a shear process. In this latter case, the ruptures were not homogeneously distributed but located in bands, indicating that the rupture went from one balloon to its neighbour.

\section{Fig.11 (double)}

At this stage acoustic emission events cannot be directly related to balloon microstructures, however they confirm similar trends than results obtained previously by other techniques. Acoustic emission brings additional improvement in the understanding of the behaviour of syntactic foam under pressure. As it is an in-situ method, it is possible to continuously record the events and then detect early damage appearance, which is not possible with X-Ray tomography because this technique requires time to make a scan and then the mechanical test must be interrupted. Fig. 12 presents a zoom of the first part of compression curve on PP foam. This enlargement shows that the acoustic emission begins around $2000 \mathrm{~N} \mathrm{(25} \mathrm{MPa).} \mathrm{There} \mathrm{are} \mathrm{obviously} \mathrm{very} \mathrm{few} \mathrm{events} \mathrm{at} \mathrm{this}$ 
loading, but this means that some degradation will be present in the material since the installation at 3000 meters water depth. In addition, the early breakage concerns the large diameter microballoons, which are of primary interest for the insulation and buoyancy capabilities of the material [17].

Fig.12 (simple)

\section{Conclusion}

The use of polymer based syntactic foams reinforced with glass microballoons for pipeline insulation in deep water depths requires a better understanding of their behaviour. Following good ageing results in relative humidity, the influence of top layer on industrial structure was studied using foam samples embedded in pure polymer. The water absorption curves coupled with modelling of the samples showed that despite the protection of the coating, the foam ages as in liquid water. There is no improvement of the ageing resistance of the foam.

Modelling of the insulated structure revealed that the solicitation of the coating is non homogeneous. A confined compression set-up was used to simulate this kind of loading. Coupled with this mechanical test, acoustic emission monitoring leads to compare various syntactic foam materials. Soft matrices present continuous and uniform damage whereas brittle resins like epoxy withstand higher load but induce sudden and localised damage. Thanks to continuously recording of acoustic emission it is possible to detect early damage related to the first breaks of glass microballoons. As lots of different phenomena occur in syntactic foams during hydrothermal ageing, acoustic emission seems to be a powerful technique to study and discriminate the evolution of degradation processes during ageing.

\section{References}

1. MATHIEU Y., IFP forum, September 2006

2. AYERS R.R., COLLERAIN J.B., Deepstar CTR 6301, october 2004

3. ROUILLON J., OTC 14171, Proceedings of Offshore Technology Conference, Houston, May 2002

4. OKONKWO U., URALIL F., BERTI E., JUDD S., Proceedings of Deep Offshore Technology, Vittoria, November 2005

5. KOTCHEKOV V.A., MAKSIMOV R.D., J. Mater. Sci. 32-1 (1996), 61-70

6. SAUVANT-MOYNOT V., GIMENEZ N., SAUTEREAU H., J. Mat. Sci, 47 (2006), 4047-4054

7. GIMENEZ N. SAUVANT-MOYNOT N., SAUTEREAU H., Proceedings of Offshore Mechanics and Artic Engineering, Halkidiki, 2005

8. CHOQUEUSE D., CHOMARD A., CHAUCHOT P., Proceedings of Offshore Technology Conference, Houston (2004) OTC16503

9. CHOQUEUSE D., CHOMARD A., BUCHERIE C., Proceedings of Offshore Technology Conference, Houston (2002) OTC14115

10. WANG W.T., WATKINS L., Proceeding of Offshore Mechanics and Artic Engineering, Halkidiki, 2005

11. HALDANE D., VAN DER GRAAF F., LANKHORST A.M., Proceedings of Offshore Technology Conference, Houston (1999) OTC11040

12. BOUCHONNEAU N., SAUVANT-MOYNOT V., GROSJEAN F., CHOQUEUSE D., PONCET E., PERREUX D., Proceedings of Offshore Technology Conference, Houston (2007) OTC1879

13. LEKATOU A.; FAIDI S.E.; GHIDAOUI D.; LYON S.B.; NEWMAN R.C., Comp; PartA, 28 (1997), 223-236

14. VERDU J., Techniques de l'Ingenieur AM3165

15. CARTER H.G. et KIBLER K.G, J.of Comp. Mat., 1978, 12, 118-131

16. SAUVANT-MOYNOT V., SCHWEITZER S., GRENIER J., DUVAL S., Proceeding of EUROCORR 2004, (Nice, France, Sept 2004). 
17. J. ADRIEN, E. MAIRE, N. GIMENEZ, V. SAUVANT-MOYNOT, Act. Mat.55 (2007), 16671679

18. D.CHOQUEUSE, D.PERREUX, V.SAUVANT-MOYNOT, J. Mat. Sci, submitted

\section{Figures}

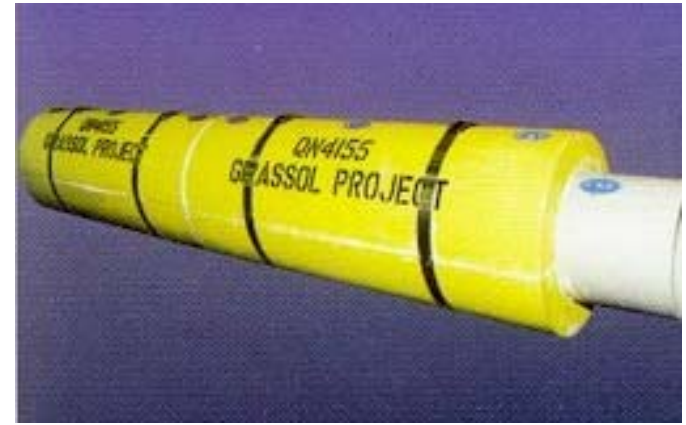

Fig. 1 Insulation modules system (CRP doc)

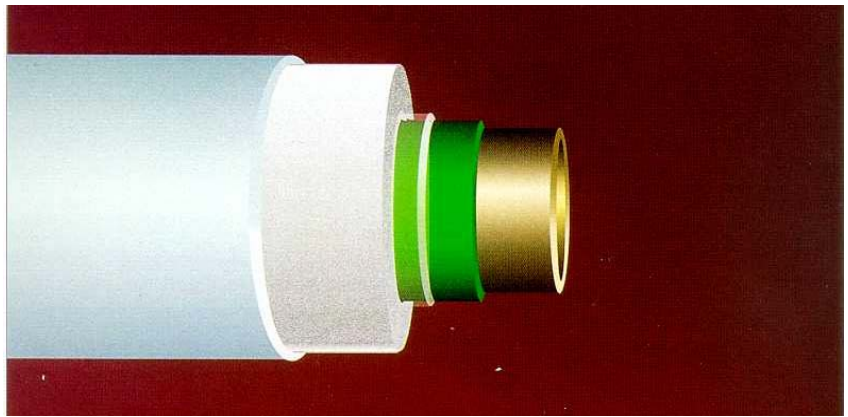

Fig. 2 Five layer insulation system (Socotherm)

\section{Epoxy coat}

Epoxy syntactic foam

Fig. 3 Bi-layer samples

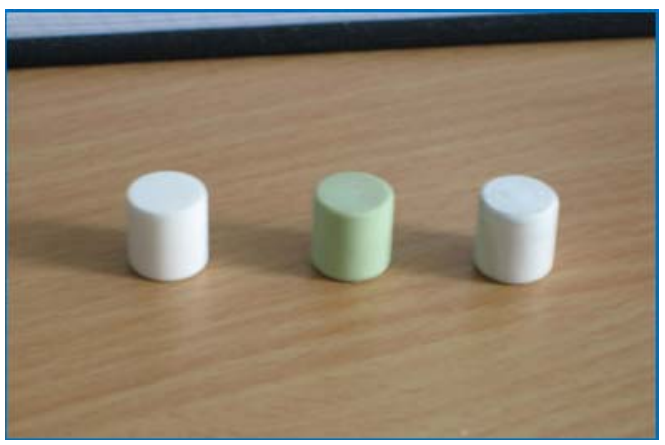

Fig. 4 Syntactic foam samples for compression test 

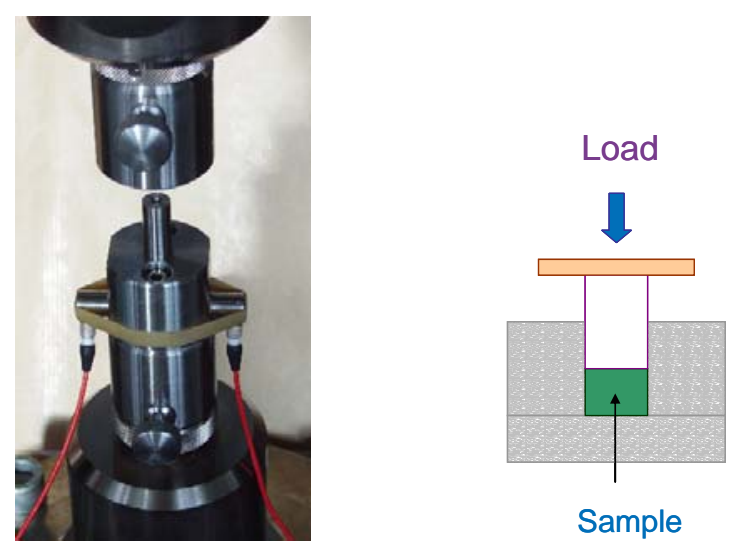

Fig. 5 Confined compression set-up

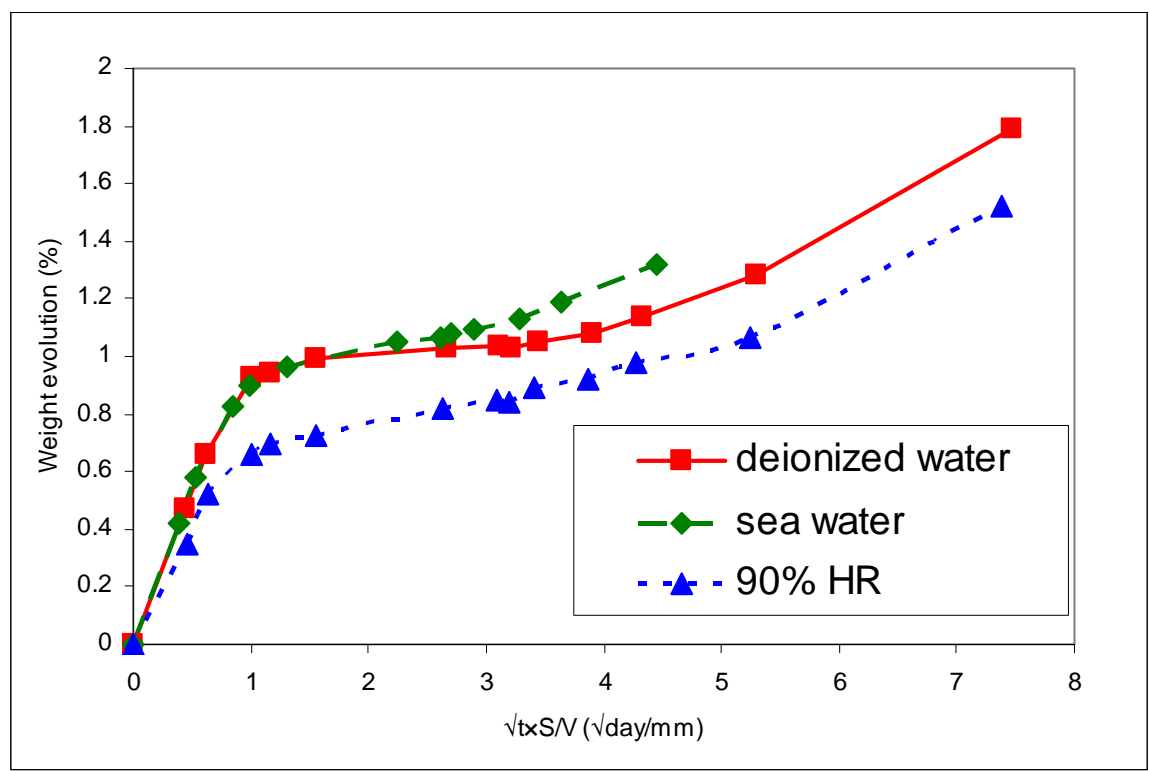

Fig. 6 Weight gains comparison of pure epoxy-anhydride aged in three environments

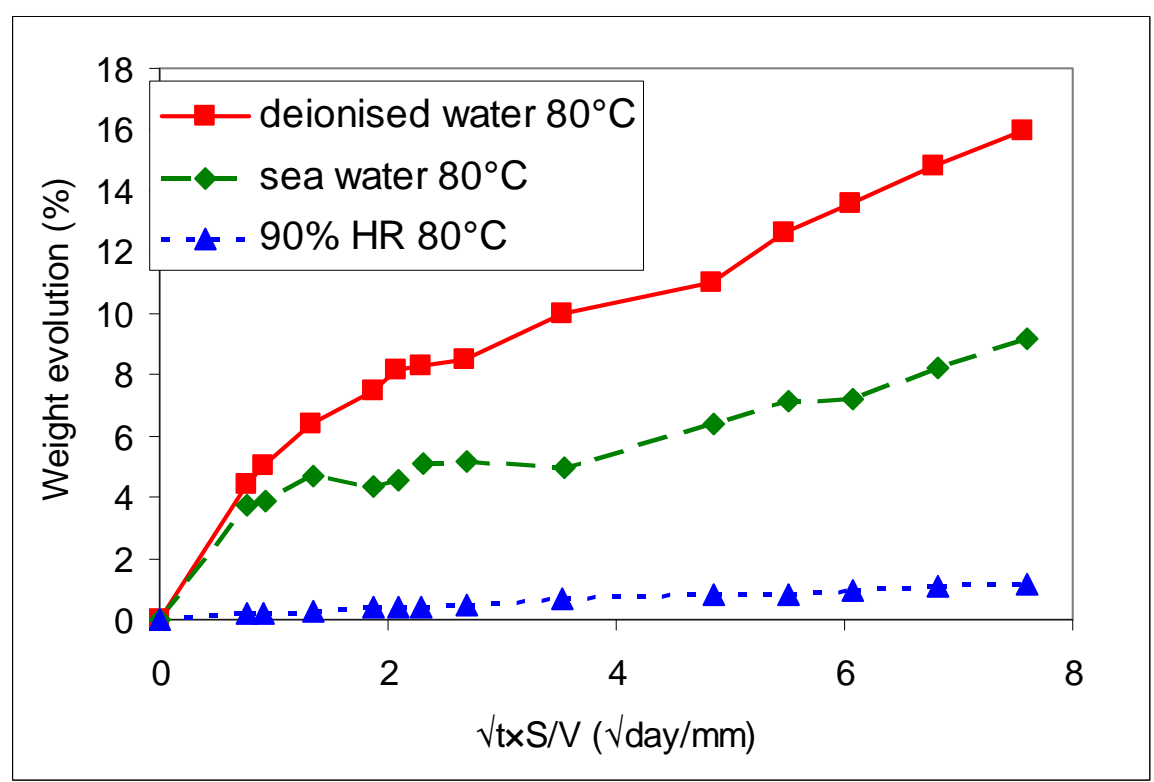

Fig. 7 Weight gains comparison of epoxy syntactic foam aged in three environments 


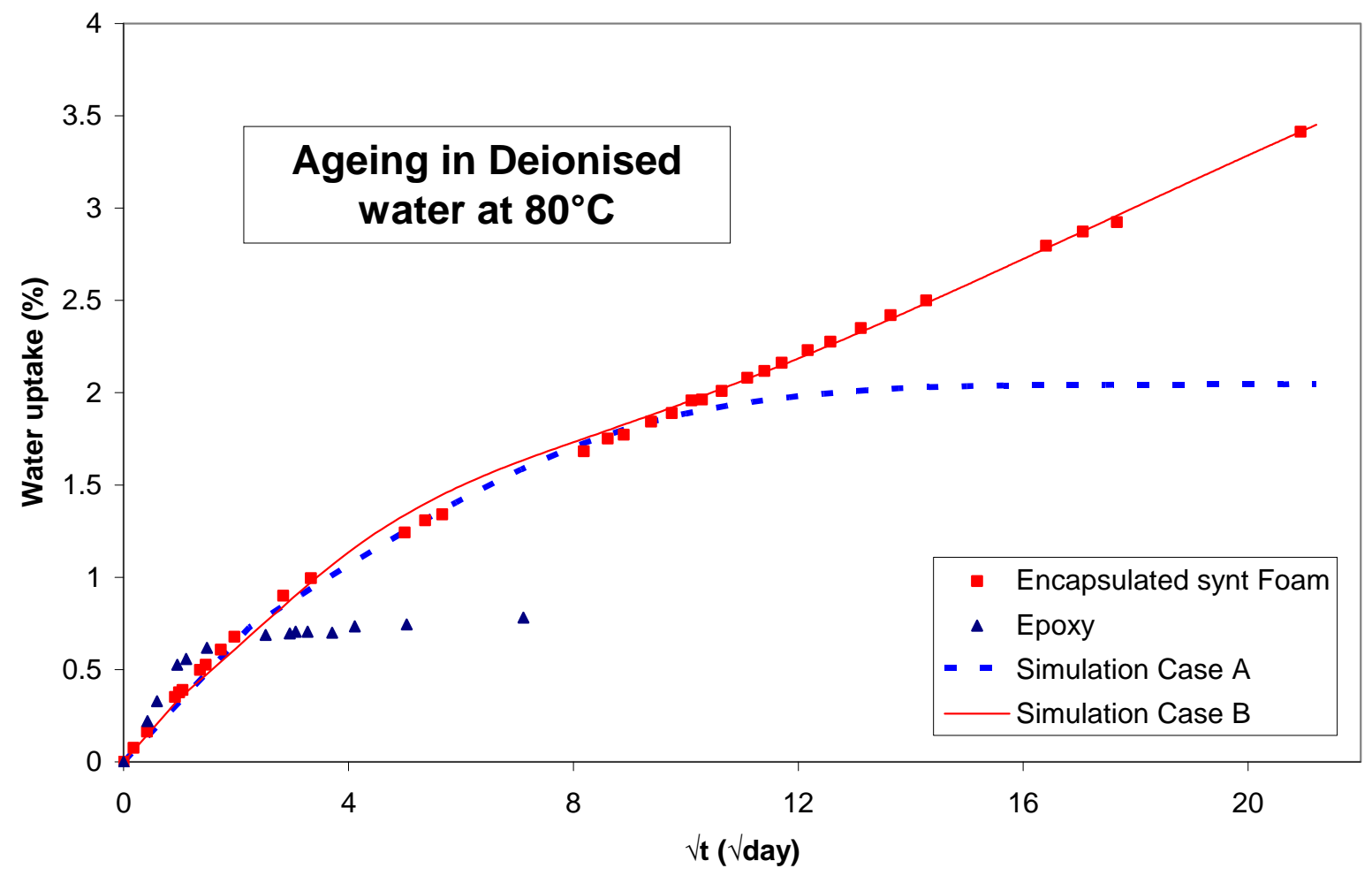

Fig. 8 Comparison of the experimental and simulated weight gains of bi-layer samples Two simulated combinaison of hypotheses for bi-layer sample: Case A: Fick diffusion on water in epoxy and Fick diffusion of relative humidity in foam Case B: Fick diffusion on water in epoxy and Langmuir diffusion of water in foam

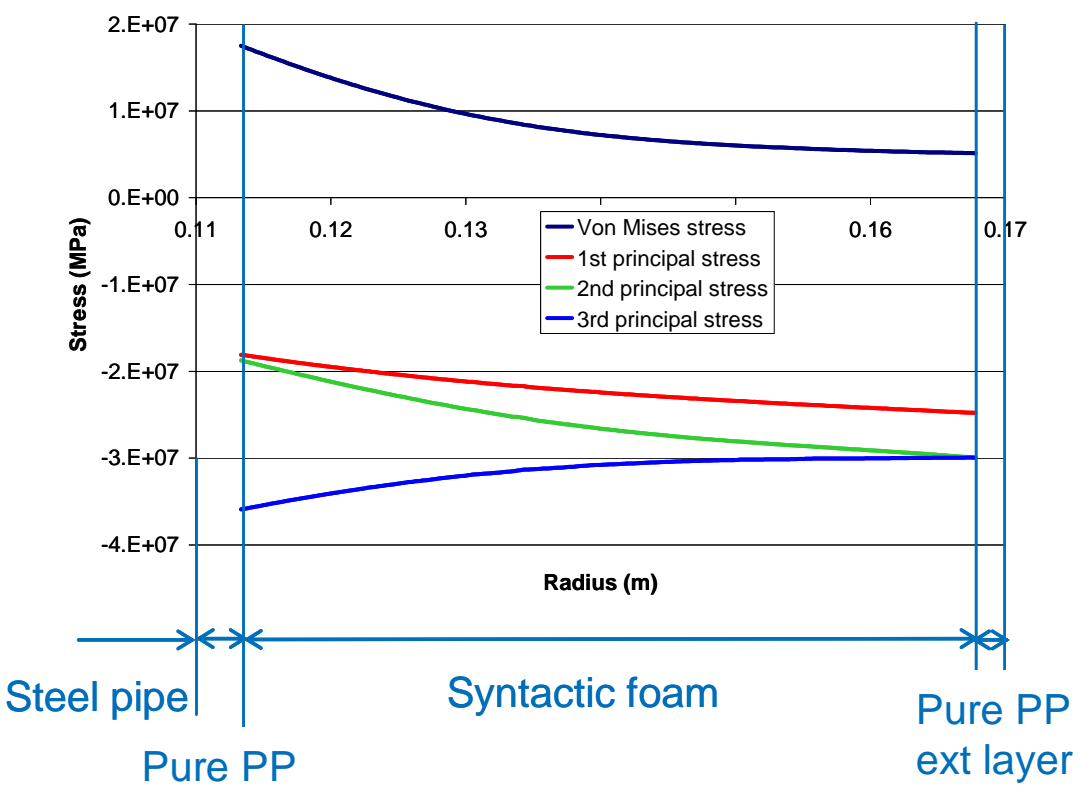

Fig. 9 Simulated stresses repartition in the syntactic foam when the insulated pipe is submitted to $30 \mathrm{MPa}$ hydrostatic pressure 


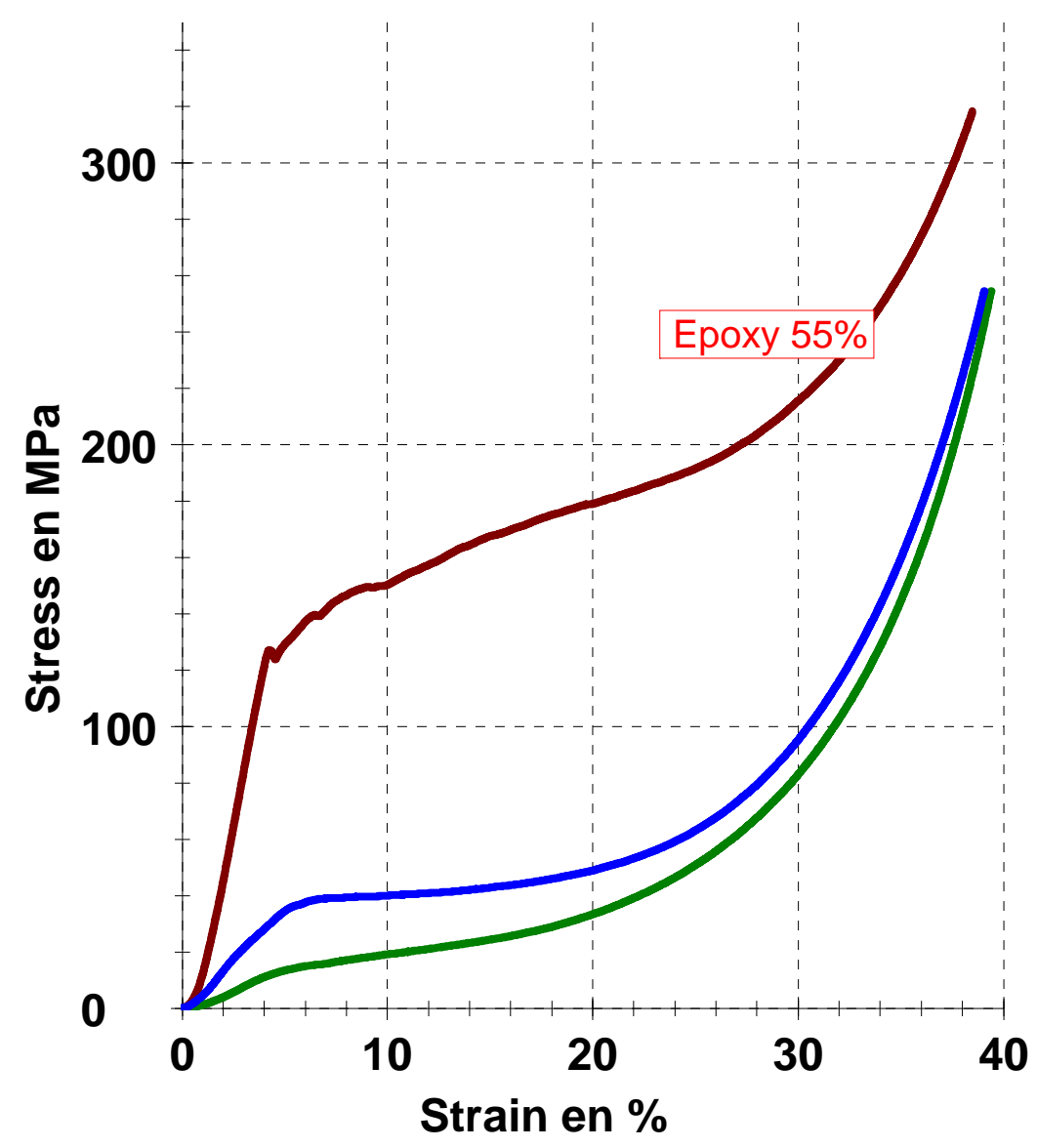

Fig. 10 Confined compression curves syntactic foams
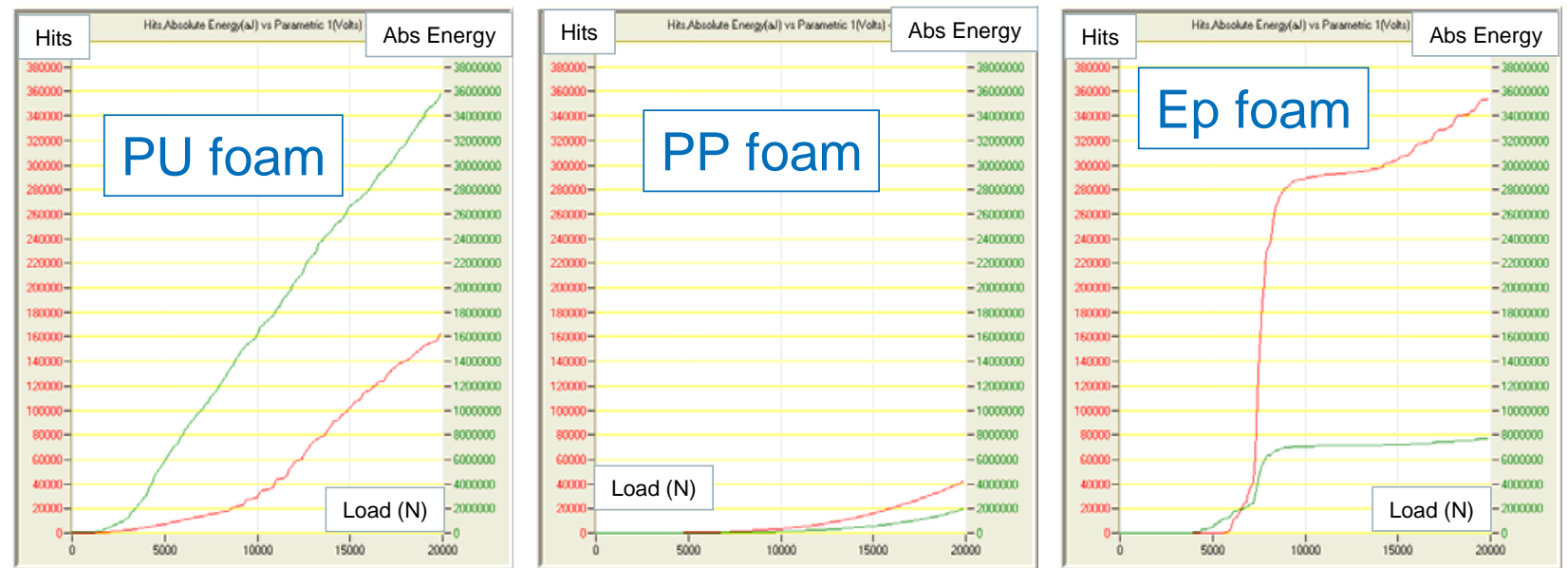

Fig. 11 Evolution of cumulative number of hits (red) and Absolute energy (green) versus applied load for the three kinds of syntactic foams 


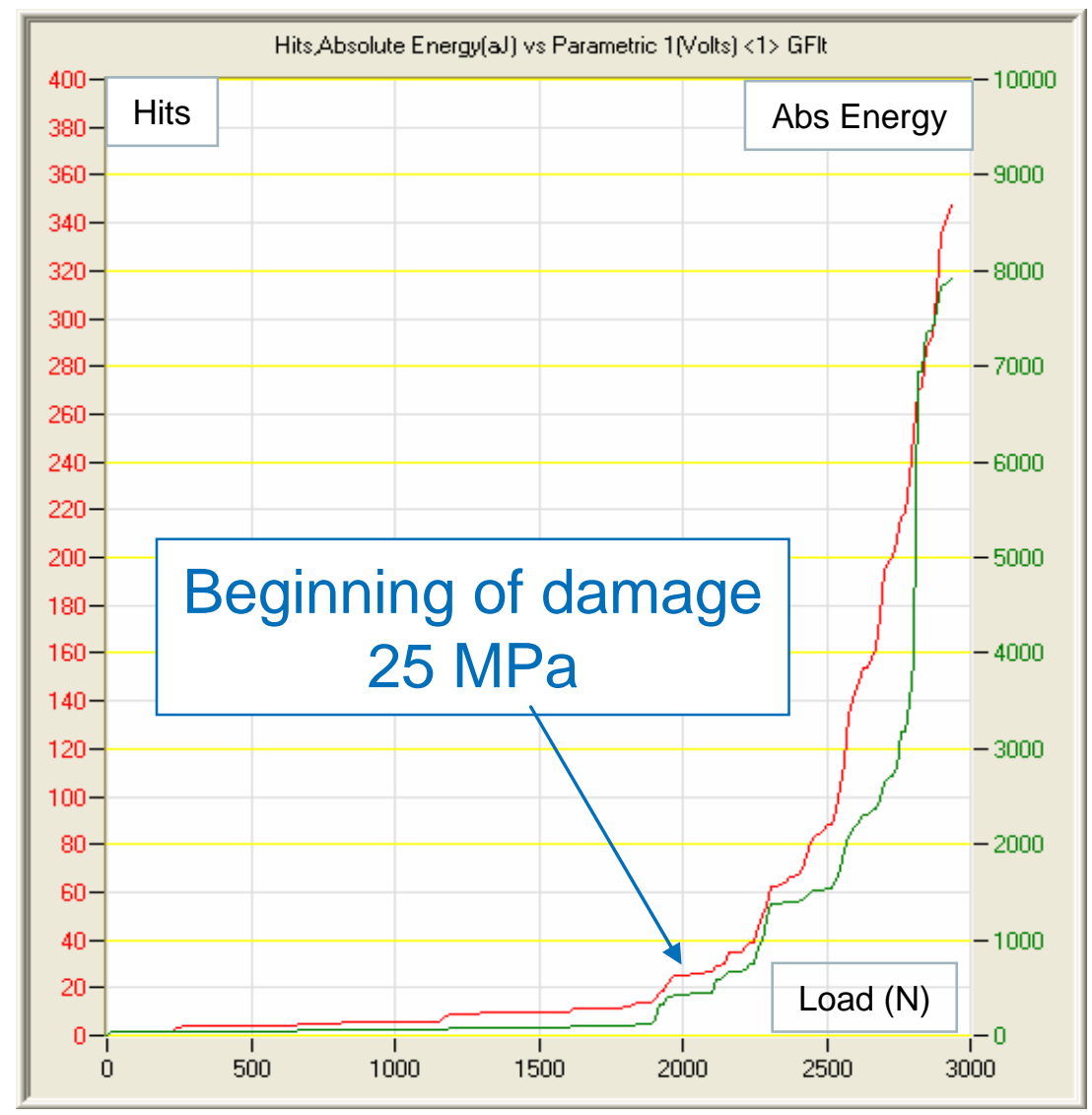

Fig. 12 Detection of the early damage on PP syntactic foam 